\title{
THE GIVE AND TAKE GAME: ANALYSIS OF A RESOURCE SHARING GAME
}

\author{
PEDRO MARIANO $^{a, *}$, LUÍS CORREIA $^{a}$ \\ ${ }^{a}$ BioISI-Biosystems \& Integrative Sciences Institute, Faculty of Sciences \\ University of Lisbon, Campo Grande, 1749-016 Lisbon, Portugal \\ e-mail: \{plmariano, luis.correia\}@ciencias.ulisboa.pt
}

\begin{abstract}
We analyse Give and Take, a multi-stage resource sharing game to be played between two players. The payoff is dependent on the possession of an indivisible and durable resource, and in each stage players may either do nothing or, depending on their roles, give the resource or take it. Despite these simple rules, we show that this game has interesting complex dynamics. Unique to Give and Take is the existence of multiple Pareto optimal profiles that can also be Nash equilibria, and a built-in punishment action. This game allows us to study cooperation in sharing an indivisible and durable resource. Since there are multiple strategies to cooperate, Give and Take provides a base to investigate coordination under implicit or explicit agreements. We discuss its position in face of other games and real world situations that are better modelled by it. The paper presents an in-depth analysis of the game for the range of admissible parameter values. We show that, when taking is costly for both players, cooperation emerges as players prefer to give the resource.
\end{abstract}

Keywords: two player game, cooperation agreements, social behaviours, resource model.

\section{Introduction}

Agents do not live in isolation. They typically have to interact with others in order to solve whatever tasks they are entrusted with. This means that agents are prone to repeatedly encounter each other, which provides opportunities for free-riding or exploitive behaviours. This problem can be modelled with social games, in particular iterated games or multi-stage games, which are more adequate for this purpose. Although there is an amount of work using iterated versions of Prisoner's Dilemma (PD) (Brembs, 1996; Nowak et al., 1994), Ultimatum (Sigmund et al., 2001), Public Good Provision (PGP) (Blackwell and McKee, 2003; van Dijk et al., 2002) and various $2 \times 2$ games (Lau and Mui, 2012), these models do not present multiple strategies for cooperation. On the contrary, the multi-stage game Centipede (Rosenthal, 1981; Binmore, 1996) presents several cooperative strategies, but has a single optimal one.

Most of those games, having a single cooperating strategy, only allow the study of simple forms of cooperation; namely, players only need to take a binary decision on whether to cooperate or not. However, there are more complex forms of cooperation, such

*Corresponding author as choice of agreements (Sutter and Strassmair, 2009), and non-cooperating behaviours such as betrayal and exploitation. In such cases there is a need for games that can model multiple cooperating strategies with equivalent gains. This feature provides players with a multitude of strategy choices. Such a property is essential to support the study and development of non-trivial negotiation strategies. In games with a single cooperating strategy this is not possible.

1.1. Contributions. The drive to develop the Give and Take (Mariano and Correia, 2002a) game was the fact that most common games used to analyse cooperation either do not have multiple optimal cooperating strategies or depend on external control. We wanted an iterated game with a diversity of Nash equilibria (NEs) and Pareto optimal (PO), such that players have different options for cooperation, under their own control. Give and Take is a game where players have to share an indivisible resource, by alternating its possession. Therefore, the resource must be durable in the sense that it does not suffer significant depreciation 1 Such a game allows us to study the establishment of pre-game agreements and subsequent

\footnotetext{
${ }^{1}$ If it were not durable, there would be no opportunity for a dilemma because it would be consumed by the first player having it.
} 
possibilities of breaking it, as well as norm enforcement in a self-organised agent community. It provides an immediate parallel to a variety of social problems. As examples, we have book borrowing from a library, time sharing in a holiday facility, and expensive equipment sharing such as a community farm tractor. These problems can be solved by human populations in a self-organised way. By this, we mean that individuals sharing the resource establish their own rules to share and monitor the resource without any external intervention.

While previous work on the Give and Take game (Mariano and Correia, 2002a; 2002b; 2003) has focused on an experimental study of parameter values that favour cooperation, no theoretical analysis has yet been put forward to study the game's properties in general and how to play it in an optimal way. In this paper, we present an in-depth investigation of the possible strategy profiles for players of Give and Take, namely, NE and PO profiles under different game parameter values. The fact that, in general, there are multiple NE and PO profiles means that the best rational behaviour of a player, besides being conditioned by game parameters, is also very dependent on the behaviour of the partner. Therefore, we can foresee different ways to choose a cooperating strategy, for instance, by pre-game negotiation (Sutter and Strassmair, 2009; Anderlini, 1999) or by iterative adaptation to the opponent's strategy.

1.2. Organisation. The rest of this paper is organised as follows. In Section 2 we overview some abstract games used to analyse cooperative dilemmas, and we situate these games in relation to Give and Take. Section 3 formally describes the Give and Take game and its parameters, and presents real applications. The two subsequent sections constitute the theoretical and numerical analysis and are the major contribution of this paper. First, in Section 4 we consider the complexity of computing a NE of a Give and Take game in order to motivate our choice of strategy space, which allows us to compute the equilibria in games with an undefined number of stages. Second, in Section 5, we present numerical simulations of the equilibria with a strategy space different from the one used in the previous section. Next, in Section 6, we discuss the implications of the results previously obtained and we suggest extensions to the game. We wrap up in Section 7 with final remarks.

\section{Related work}

Many games have been used to study cooperation. Here we briefly analyse the games that are most related to Give and Take and identify the novelty that this game affords.

2.1. Resource. When we consider scenarios with an indivisible resource that must be shared, used by a single agent at a time, no other current games provide a suitable model. In general, the resource is always available for everybody, which means that in every iteration the same set of actions can be performed by any player. This is the case in PD, PGP, Ultimatum, Dictator, Centipede or Give-Or-Take-Some (GOTS) (McCarter et al., 2011), among others. In all these games, the resource is also divisible. Consequently, at any time, all the players can gain some utility from the resource.

In addition, due to these features, the resource in common games to study cooperation usually has some dynamics, externally controlled as by Nature (Akiyama and Kaneko, 2000), or by the players' actions. In PGP, the resource must be provided by the players and profitability depends on the number of providers. In Ultimatum and Dictator, the good, externally supplied, is divisible and one of the players decides how to divide it. Centipede needs an externality that makes the resource increase its value in each stage. Give and Take models a single indivisible and durable resource. In this game, payoffs are the result of using the resource.

2.2. Cooperative dilemmas. Dilemmas are present in many day-to-day situations (Fehr and Gintis, 2007; McCarter et al., 2011): Should people use public transport to favour traffic flow, or use private transportation for comfort? Should farmers over-exploit fields without any period of recovery for immediate profit, or should they give land a resting period for long term benefit? Should people pay taxes to increase government funding of public projects, or should they avoid taxes to preserve income? These are some situations where people have the option of cooperating for the common good of society, but a selfish action often gives a larger immediate payoff than a cooperating one.

There are quite a few games that model cooperative dilemmas. Three of the most commonly used are PD, its iterated version called Iterated Prisoner's Dilemma (IPD), and PGP, which can be considered an extension of PD to $n$ players. In the one-shot variant of these games, the single PO profile is dominated by other strategies, rendering a lower outcome to the game's players. Typically, one of these strategies is the single NE. These games have been used to model scenarios where a single resource must be cared for by everybody. All players have access to it and can reap its benefit. GOTS is a variant of PGP in which players can voluntarily give or take resource units from a common resource pool (McCarter et al., 2011). The strategy space of GOTS is more complex than the one of PGP, where players either provide the good or not. Other than that, in GOTS, there is no state nor do players have roles.

\footnotetext{
${ }^{2}$ We refer to Dictator because, in spite of its being a one player game, when played iteratively, players usually exchange roles.
} 
The aforementioned games are symmetric. But asymmetric ones are also used to study cooperative dilemmas. Games such as Dictator or Ultimatum have a single Nash equilibrium that is not selected as often as the theory predicts in experiments involving human subjects (Camerer, 2003). In these games, one player has a resource and must decide how to divide it with the other player. In Ultimatum, the other player decides to accept the division or not. Again, in terms of fairness, these games have a single PO profile: the resource is equally split among the two players. But other profiles are also PO in this sense. There are multi-stage variants of Ultimatum where players take turns proposing divisions of the resource (Nicolò and Yu, 2008), with players exchanging roles as determined by a third party controlling the experiment (Cason and Mui, 1998).

Centipede is also an asymmetric game with a single $\mathrm{NE}$ and a single PO, which do not coincide. It has been used to study backward induction, also with disparate results when comparing human response to theoretical rational behaviour (McKelvey and Palfrey, 1992). This game has the interesting detail of the players controlling the number of stages of the game. In each stage, a player can choose to end the game or to pass.

The existence of cooperative dilemmas and experimental results that differ from predictions has given rise to research in punishment (Ottone, 2008) and communication (Sutter and Strassmair, 2009) to promote cooperation. Some of the games previously mentioned have been extended with an additional post-game stage where players can punish their partners or with an additional pre-game stage where they communicate their strategies. Note that such extensions do not only impose an additional stage but usually generate a different NE from the original game. When the NE does not change, the addition of a punishment stage raises the question of higher order punishments, because punishment is not the rational choice. For instance, this is the case in PGP with punishment studied by Fehr and Gächter (2002) as well as Boyd et al. (2003).

In our game, punishment is built-in as it does not require a specific stage for it. One of the actions (take action) can be considered punishment. However, it may not be dominated, which contrasts with the need of second-order punishment in PGP. As we show in this article, a player may be able to recover the cost of the punishing action in the following stages.

2.3. Coordination games. Coordination games pose a different problem compared to cooperative dilemmas. Players have to coordinate their actions if they want to maximise their gains (Helbing et al., 2005). As examples of this type of games, we have Stag Hunt and Battle of Sexes. In Stag Hunt one of the pure NE is risk dominant but yields a lower payoff than the second pure NE. This constitutes a dilemma because a risk-averse player prefers the first NE. In Battle of Sexes, the two pure NE are symmetric. This is a dilemma because each player prefers the pure NE more beneficial to itself.

A coordination dilemma may be solved if players can establish an agreement or make their actions depend on some external device such as coin flipping. For instance, in Battle of Sexes, players may agree on going to the opera if the outcome is heads. Players can also resort to pre-play communication (Anderlini, 1999) as a means to solve coordination problems.

In our game, there are several equally good NE (for certain parameter values). This problem can be tackled with pre-play communication, but it can also be solved during the game. The player without the resource can observe when the other gives it away and adjust its strategy accordingly. Likewise, the player with the resource may adjust its strategy as a result of observing the other player taking it away. Give and Take contrasts with the reviewed coordination games since coordination can be obtained while playing the game.

2.4. Uniqueness of Give and Take. Besides the characteristics mentioned above, Give and Take also has unique properties of optimal equilibria and asymmetry. We shall now briefly analyse them.

PGP and PD are games with a single PO profile. When they are played iteratively, the number of NE and PO profiles may increase (see Hofbauer and Sigmund, 1998; Gintis, 2000). Centipede, Ultimatum and Dictator also have several PO profiles. However, in such games, these profiles are usually dominated by some NE. A similar situation happens when a taking-it-in-turns pattern is considered (Ward, 1998). In this case, there are typically infinite PO equilibria; however, none of them is a NE. In Give and Take, there are multiple POs that are also NEs. Therefore, in the absence of a single dominating strategy, players can choose different NEs through explicit or implicit agreements.

Give and Take is a stochastic game (SG) because resource possession influences the payoff matrices as well as players' actions. The player with the resource has a set of actions different from the set of the player without the resource. The value for a given action is not the same in the two sets. Moreover, in each stage, players' actions control the role they have in the subsequent stage of the game. This is uncommon since in other known asymmetric games, such as iterated Centipede or Ultimatum, the players change roles by external control.

Several characteristics of real problems are not captured by the games we have reviewed, which are among the most widely used in social and economic sciences. The metaphor of a library book, or a community tractor, is better modelled with the Give and Take game rather than with PD, PGP, Dictator, Ultimatum or their 
iterated versions, or Centipede. To the best of our knowledge, Give and Take is the most adequate game to model self-organised sharing of a durable resource through time. The existence of multiple coincident NE and PO profiles provides an increased realism to model social dilemmas, where players usually have a varied set of cooperating options available.

\section{Description of Give and Take}

We now describe Give and Take and the dilemma that players are faced with.

3.1. Overview. This game has an unbalanced nature since players have different roles depending on who holds the resource at the beginning of every stage. Initially, a random player is assigned the possession of the resource, which is indivisible and durable. The player with the resource has a benefit from using it. Actions available to a player depend on its role. There are two actions for each player, one of them being identical for both. The player with the resource can give it, give $(\mathrm{G})$, to its partner, who always accepts it; the player without the resource can forcefully take it, take (T); and both players can do nothing - we may distinguish this case as none ${ }_{r}(+)$ and none $_{\neg r}(-)$ for the player with the resource and the player without the resource, respectively. If a player gives the resource away, it receives a bonus. A player that takes the resource pays a penalty as does its partner, although these penalties may be different. This is a multi-stage game, where the number of stages is externally determined, if limited. Each discrete time instant corresponds to a stage. Players' roles remain the same, provided both players do nothing. Players exchange roles whenever the player with the resource gives it or the player without the resource takes it.

3.2. Formalisation. Give and Take can be represented as a SG (Shapley, 1953). The state of the SG represents who has the resource. This means the set of games contains two elements 3 The set of actions for the player with the resource is $S_{r}=\left\{\right.$ none $_{r}$, give $\}$, while the set of actions for the player without the resource is $S_{\neg r}=$ $\left\{\right.$ none $_{\neg r}$, take\}. The state transition probability function is either zero if both players play the none actions, or one otherwise.

Give and Take has three parameters which are related to the game actions: $b_{g}$ is the bonus received by the performer of the give action, $c_{p t}$ is the cost paid by the take action performer, and $c_{s t}$ is the cost paid by the take action subject. The parameter values are analysed in Sections 3.4 and 4 . The resource possession, $p_{r}$, is

\footnotetext{
${ }^{3}$ We use the definition of the SG by Shoham and Leyton-Brown (2009).
}

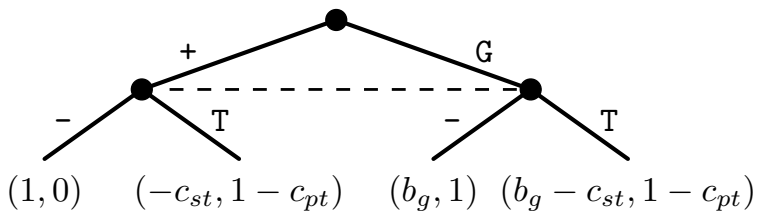

Fig. 1. Extensive form description of a stage of Give and Take. The first information set (counting from the top) represents the player with the resource, $r$, while the second information set the player without the resource, $\neg r$. The first value of the payoffs pair is for the player with the resource. See the text for an explanation of the payoff values.

one unit and this possession is accounted for after both players' actions. This means that, if both players do nothing, the player with the resource gains one unit and the other gains nothing. Figure1 1 shows the extensive form of one game stage. From this figure we can extract the stage payoff matrices.

Since the game stage is asymmetric, there are two stage payoff matrices. Each payoff matrix is characterised by a subscript representing who has the resource, $r$, and who does not have it, $\neg r$. In both matrices, each row corresponds to a different action of the player with the resource and each column corresponds to a different action of the player without the resource. Namely, the top row and left column correspond to action none of the two players, the bottom row corresponds to action give (player with the resource), and the right column corresponds to action take (player without the resource). The payoff matrix of the player with the resource is

$$
A_{r}=\left[\begin{array}{cc}
1 & -c_{s t} \\
b_{g} & b_{g}-c_{s t}
\end{array}\right],
$$

and the payoff matrix of the player without the resource is

$$
A_{\neg r}=\left[\begin{array}{cc}
0 & 1-c_{p t} \\
1 & 1-c_{p t}
\end{array}\right] .
$$

The asymmetry of this game is clear as the matrices are not equivalent, i.e., the matrix in Eqn. (1) is not the transpose of the matrix in Eqn. (2).

Figure 2 depicts the stochastic game behind Give and Take with an undefined number of stages. We consider that Nature defines the initial state by assigning the resource to one of the players.

3.3. Payoff computation. Consider the following action sequence where time goes from left to right:

$$
\begin{array}{|l|}
\hline+\mathrm{G}--+\mathrm{G}--+\mathrm{G}--++--++--++\mathrm{T}++\mathrm{T} \\
--+\mathrm{G}--+\mathrm{G}--+\mathrm{G}-\mathrm{T}+\mathrm{G}-\mathrm{T}+\mathrm{G}-\mathrm{T}+-\mathrm{T}+
\end{array} .
$$

Clearly, the top player started with the resource. An action sequence can be divided into periods where each period is 


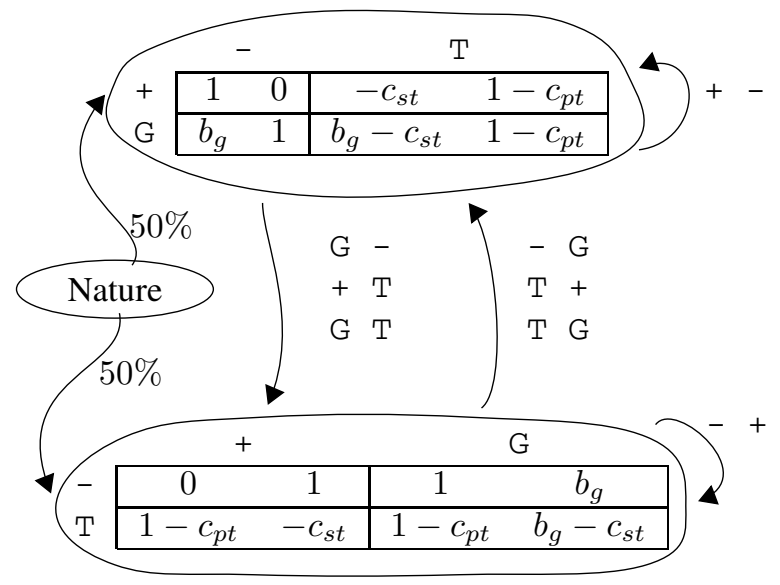

Fig. 2. Graphical representation of the stochastic game and Nature's initial move in Give and Take. Nature initially gives the resource either to the row player (top table) or to the column player (bottom table). Each table represents a game of the stochastic game. The arrows that depart from these tables represent the transition probability function. The transition is deterministic and an arrow is labelled with an action profile of the departing game.

characterised by the actions performed by each player. In particular, the example can be split in three periods:

\begin{tabular}{|c|c|c|}
\hline$+\mathrm{G}--+\mathrm{G}--+\mathrm{G}--$ & ++--++-- & $++\mathrm{T}++\mathrm{T}$ \\
$--+\mathrm{G}--+\mathrm{G}--+\mathrm{G}$ & $-\mathrm{T}+\mathrm{G}-\mathrm{T}+\mathrm{G}$ & $-\mathrm{T}+-\mathrm{T}+$ \\
\hline
\end{tabular}

All the three periods have different action sequence patterns. In the first period the players only perform the give action. Here the action sequence pattern that repeats is one player performing the give action in some stage, and afterwards the other player also performs the give action. In the second period, the top player only plays the none actions while the bottom player takes and gives the resource. In the last period, players only play the take action.

There are six possible action sequence patterns, which are shown in Table 1. Each pattern was assigned a class; for instance, in Class $\mathcal{A}$ both players give the resource. Some action sequences may belong to the same class but differ on the number of stages that players take to perform an action. Each class has two specific payoff expressions (one for each player) that are a function of the number of stages, $t_{r}$ (a player holds the resource before giving it or having it taken), and the number of stages, $t_{\neg r}$ (it waits without the resource before taking it or being given it). Note that these two parameters are not a characterisation of a player's strategy. They are rather a characterisation of an action sequence pattern.

We can augment the example that we presented in the beginning of this section with the action sequence pattern classes:
Table 1. Classification of the six action sequence patterns. Time goes from left to right. The third column contains the value of variables $F_{k}^{t}$ and $F_{k}^{b}$ used in the top and bottom players' payoff, respectively (see Eqn. (3)).

\begin{tabular}{|c|c|c|}
\hline Class & Action sequence pattern & $F_{k}^{t} F_{k}^{b}$ \\
\hline $\mathcal{A}$ & $+\cdots+G-\cdots-+$ & $b_{g}$ \\
\hline \multirow{2}{*}{$\mathcal{B}$} & $+\cdots+\mathrm{G}-\cdots-\mathrm{T}$ & $b_{g}-c_{p t}$ \\
\hline & $-\cdots-++\cdots+G$ & $b_{g}-c_{s t}$ \\
\hline \multirow{2}{*}{$\mathcal{C}$} & $+\cdots+\mathrm{G}-\cdots-\mathrm{T}$ & $b_{g}-c_{p t}$ \\
\hline & $-\cdots-++\cdots+-$ & $-c_{s t}$ \\
\hline \multirow{2}{*}{$\mathcal{D}$} & $+\cdots+\mathrm{G}-\cdots-\mathrm{T}$ & \multirow{2}{*}{$b_{g}-c_{p t}-c_{s t}$} \\
\hline & $-\cdots-\mathrm{T}+\cdots+\mathrm{G}$ & \\
\hline \multirow{2}{*}{$\mathcal{E}$} & $+\cdots+\mathrm{G}-\cdots-\mathrm{T}$ & $b_{g}-c_{p t}-c_{s t}$ \\
\hline & $-\cdots-\mathrm{T}+\cdots+-$ & $-c_{p t}-c_{s t}$ \\
\hline $\mathcal{F}$ & $\begin{array}{l}+\cdots+-\cdots-\mathrm{T} \\
-\cdots-\mathrm{T}+\cdots+-\end{array}$ & $-c_{p t}-c_{s t}$ \\
\hline
\end{tabular}

\begin{tabular}{|cccccccc}
$\mathcal{A}$ & $\mathcal{A}$ & $\mathcal{A}$ & $\mathcal{C}$ & $\mathcal{C}$ & $\mathcal{F}$ & $\mathcal{F}$ \\
\hline$+\mathrm{G}--$ & $+\mathrm{G}--$ & $+\mathrm{G}--$ & ++-- & ++-- & $++\mathrm{T}$ & $++\mathrm{T}$ \\
$--+\mathrm{G}$ & $--+\mathrm{G}$ & $--+\mathrm{G}$ & $-\mathrm{T}+\mathrm{G}$ & $-\mathrm{T}+\mathrm{G}$ & $-\mathrm{T}+$ & $-\mathrm{T}+$ \\
\hline
\end{tabular}

The periods shown in the last sequence were further divided at the stage where the resource went back to the top player. With this division, class $\mathcal{A}$ action sequence pattern repeats three times. During each sub-period, the top player kept the resource during $t_{r}=2$ stages and spent $t_{\neg r}=2$ stages without the resource. The action sequence pattern in the fourth and fifth sub-periods belongs to class $\mathcal{C}$. In this class, only the bottom player performed the give and take actions. In the last two sub-periods, which belong to class $\mathcal{F}$, the top player had the resource during $t_{r}=2$ stages and spent $t_{\neg r}=1$ stage without the resource.

From the matrices (1) and (2) we deduce players' payoff. Analysing any of the six action sequence patterns presented in Table 1, their duration is $t_{r}+t_{\neg r}$. During this time the top player holds the resource for $t_{r}$ stages, which earns it $t_{r}$ payoff units. However, we also need to take into account the actions performed by both players. Let $F_{k}^{t}$ be an expression depending on $b_{g}, c_{p t}$ and $c_{s t}$. The average payoff obtained per game state by the top player in class $k \in\{\mathcal{A}, \ldots, \mathcal{F}\}$ is

$$
u_{k}^{t}=\frac{1}{t_{r}+t_{\neg r}}\left(t_{r}+F_{k}^{t}\right),
$$

where the value of $F_{k}^{t}$ is given in the third column of Table 1. A similar expression gives the payoff for the bottom player. We need to swap the $t_{r}$ and $t_{\neg r}$ parameters and use the $F_{k}^{b}$ parameter. Whenever both players play the same set of actions, which occurs in classes $\mathcal{A}, \mathcal{D}$ and $\mathcal{F}$, only one value of $F_{k}$ is shown. For any finite game, the 
average payoff is a weighted average of $u_{k}$,

$$
\frac{\sum_{k} w_{k} u_{k}}{\sum_{k} w_{k}}
$$

where $w_{k}$, the weight of $u_{k}$, is the number of times class $k$ occurred during the game.

3.4. Dilemma setup. Considering that parameters $b_{g}$, $c_{p t}$ and $c_{s t}$ are non-negative, we now focus our attention on their upper bounds and on the limit case of zero value.

Parameter $b_{g}$. If $b_{g}$ is greater than one, the player with the resource is better off if it gives it to its partner. Its payoff matrix is reduced to a $1 \times 2$ vector because action none $_{r}$ is dominated by action give. Since the resource is going to exchange hands, should the partner regain it? As $c_{p t}$ is a non-negative constant, it should do nothing, waiting to be given the resource. Summarising, the payoff matrices (1) and (2) are reduced to row give and column none $_{\neg r}$. After eliminating dominated strategies, the Nash equilibrium is $\sigma=(1,0)$. This means that, independently of the (even) number of stages that are going to be played, the only optimum strategy profile in this case is, in every stage: the player with the resource gives it and the other does nothing (action none $\neg_{\neg r}$ ). For any number of stages, the approximated average payoff per stage is

$$
\frac{1+b_{g}}{2} \quad \text { if } \quad b_{g} \geq 1
$$

In this case, there is no dilemma, as the rational choice is also PO. Players are better off giving away the resource in every stage. This is no surprise since $b_{g} \geq 1$ means that the gain of giving the resource is larger than of keeping it. Therefore, from now on, we shall focus on $b_{g}<1$. With $0 \leq b_{g}<1$, we have a dilemma in this game.

The limit case of $b_{g}=0$ does not change dominance in the single stage payoff matrices. However, a different dilemma arises when there are multiple stages. The resource can be exchanged at different time rates, provided they are identical for both players, without changing the players' payoffs. In this case, there is a coordination dilemma.

Parameter $c_{p t}$. If $c_{p t}$ is zero, taking the resource is free and action take weakly dominates action none $\neg r$. As for the player with the resource, knowing this, it should give the resource. This means that, independently of the (even) number of stages that are going to be played, the only optimum strategy profile in this case is, in every stage: the player with the resource gives it and the other takes it. For any number of stages, the approximated average payoff per stage is

$$
\frac{1+b_{g}-c_{s t}}{2} \quad \text { if } \quad c_{p t}=0
$$

This parameter does not have an upper bound. It can grow indefinitely without introducing dominating strategies in the single stage payoff matrices.

Parameter $c_{s t}$. This parameter by itself does not have an influence on dominance and equilibria in the single game stage.

Parameter domain. Combining all these results, the complete parameter domain is

$$
\left(b_{g}, c_{p t}, c_{s t}\right) \in[0,1[\times] 0, \infty[\times[0, \infty[.
$$

3.5. Applications of Give and Take. We now dwell on the interpretation of the Give and Take game. The applications better modelled by this game are cases of self-organised sharing of durable goods. In those cases, if someone currently holding the resource does not give it back to its partner after some reasonable time, it may be punished by the partner. However, enforcing a penalty is usually costly. In real social situations, such a process takes time and effort for both the performer and the subject involved, whether the penalty is directly inflicted by a partner or by a public entity. This is captured by parameters $c_{p t}$ and $c_{s t}$.

The bonus, even if not material, can be interpreted at least as a reputation side effect, with some equivalent value, typically less than the benefit of using the resource. This means that, in real cases modelled, when there is no material bonus for giving the resource, we assume that there is a positive social impact for the person who gives it. With this in mind, we shall now revisit the examples given in Section 1.1 in greater detail.

Suppose that two farmers share a tractor. Each may use it for some limited time, taking profit $p_{r}$ from it in plowing the land. After that, it may voluntarily yield the tractor, which is positively evaluated by its partner in a subjective way, $b_{g}$. Otherwise, it may risk a forceful action from the partner. In this process, the farmer that recovers the resource and the one that did not relinquish it may suffer some kind of loss, $c_{p t}$ and $c_{s t}$ respectively, not necessarily identical for both.

In the case of borrowing a book from a library, $p_{r}$ can be the subjective value of the pleasure or utility a reader gets from reading a book. If individual $B$ returns a book with delay, the time reader $A$ waits for the book is $c_{p t}$ and a period of inhibition imposed on $B$ is $c_{s t}$. In this case, $b_{g}$ may be a contribution for the reliance of a reader. A low or null reliance may aggravate the inhibition period, while a positive reliance does not produce such an effect. Therefore, $c_{s t}$ may depend on the reader's reliance.

A holiday house tends to be disputed in high season. Its usage for a period may be evaluated as benefit $p_{r}$ and to relinquish it on time contributes $b_{g}$ to a subjective positive 
reputation. If one must evict an abuser, it incurs a cost $c_{p t}$, economically and in discomfort, while the abuser suffers a penalty $c_{s t}$, comprising a possible inhibition of use.

A service contract may also be easily modelled by Give-Take. If both sides are happy with the result, it is equivalent to assume that both alternately perform the give action. Periodically one provides a service and the other pays for it, involving a cost/benefit of $p_{r}$. Here, $b_{g}$ can also be considered a positive contribution to a subjective reputation. If one of the partners, say $A$, is not satisfied with the contract, it may claim nonfulfillment. $A$ pays some litigation cost, $c_{p t}$, and may recover the contracted deal. The other partner, $B$, gets a penalty, $c_{s t}$, for not providing the service.

\section{Theoretical analysis of equilibria}

The problem of computing a NE of a two-player general-sum game is NP-complete (Chen and Deng, 2006). In particular, this problem belongs to the class PPAD, or the polynomial parity argument, directed version (Papadimitriou, 1994). This means the time to compute this problem is exponential in the size of the game; in particular, in the size of the strategy space.

Backward induction in finite games is used to reduce the strategy space, and thus the complexity of computing a NE. If we apply backward induction, the reduction in strategy space is meager. In the last $\left\lceil c_{p t}\right\rceil$ stages both players do nothing: the player without the resource is not able to recover the cost of the take action, and the player with the resource has no incentive to give it. In the real world scenarios given in Section 3.5, the number of stages $l$ may be much greater than $c_{p t}$. In the end, backward induction is not very helpful.

The computation of a NE of a game in extensive form is exponentially faster compared to a game in normal form (Koller et al., 1996). In particular, in Give and Take it is $2^{l}$ versus $2^{2^{l}-1}$. Even if we used the extensive form of a finite Give and Take game (built using the tree in Fig. 1), the number of information sets is exponential in $l$. Thus, there is an exponential number of constraints in the corresponding linear complementary program (Shoham and Leyton-Brown, 2009).

To the best of our knowledge, there is no efficient method to compute a NE of Give and Take with a finite or infinite number of stages. Regarding the finite game, the procedure belongs to the class PPAD. Backward induction does not considerably reduce the strategy space. As for the infinite game, it is not possible to model Give and Take as a repeated game (for which it is possible to compute a NE).

In this section, we consider a specific strategy space that allows us to draw some properties regarding how players using strategies from this space would play Give and Take. The analysis focuses on games with an infinite number of stages.

4.1. Strategy space. Strategies prescribe the action a player should play for every history. Here we restrict the game analysis to pure strategies with only two parameters: $t_{g}$, the number of stages a player holds the resource before giving it, and $t_{t}$, the number of stages a player waits without the resource before taking it. This defines an $\mathbb{N} \times \mathbb{N}$ strategy space. This space, with constant $t_{g}$ and $t_{t}$, is the simplest pure strategy space of Give and Take.

While our choice may look too simple, we must realise that, with contingent strategies, the strategy space grows exponentially with history size. Therefore, a choice must be made on how much history a strategy can handle. Hofbauer and Sigmund (1998) used a strategy, when analysing the IPD game, that only takes into account what happened in the last iteration. With our choice of strategy space, a Give and Take player, besides representing $t_{g}$ and $t_{t}$ to specify the strategy, must record a time value. The latter is countable (discrete time), representing how long ago the resource last changed hands. Notice that the strategies in this strategy space are minimally contingent because players only need to record the time of the last resource movement. Pure non-contingent strategies are the trivial cases with $\left(t_{g}, t_{t}\right) \in\{1, \infty\} \times\{1, \infty\}$. In such cases, a player never gives the resource or gives it right away when it receives it, and likewise for the take action. There is no need for history representation.

The proposed strategy space, although simple, allows us to obtain rich interaction dynamics (see the following sections). In this first analysis of Give and Take, we need to establish the results of the simplest strategies in the game. The insight gained will also prove useful for future developments of dynamical strategy spaces.

4.2. Classes of strategy pairs. We are going to characterise strategy profiles, which are pairs of strategies $\left(\mathfrak{s}_{i}, \mathfrak{s}_{j}\right)$, with $\mathfrak{s}_{i}=\left(t_{g}^{i}, t_{t}^{i}\right)$ and $\mathfrak{s}_{j}=\left(t_{g}^{j}, t_{t}^{j}\right)$, for players $i$ and $j$. The characterisation is based on relations $t_{g}^{i} \gtreqless t_{t}^{j}$ and $t_{t}^{i} \gtreqless t_{g}^{j}$. Each one of the nine combinations produces one of the six action sequence patterns presented earlier in Section 3.3 Thus, the characteristic of a strategy profile is the class of the action sequence pattern. Table 2 shows these characteristics but considers players unidentifiable (meaning it is indifferent which player performs each action sequence). For example, we say that the pair of strategies $\mathfrak{s}_{i}=(1,3)$ and $\mathfrak{s}_{j}=(2,3)$ belongs to class $\mathcal{A}$. Each pattern is repeated with a period equal to its length (shown in third column of Table 2).

The payoffs of the two players, top and bottom in each class in Table 1, may be different. In classes $\mathcal{B}, \mathcal{C}$ and $\mathcal{E}$, this is clear since the action sequences of the two players are different. In classes $\mathcal{A}, \mathcal{D}$ and $\mathcal{F}$, the payoffs can be different when $t_{g}^{i}$ and $t_{g}^{j}$ differ. 
Table 2. Characterisation of profiles based on strategies $\left(t_{g}^{i}, t_{t}^{i}\right)$ and $\left(t_{g}^{j}, t_{t}^{j}\right)$. Each profile has an action sequence pattern periodically repeated.

\begin{tabular}{|c|l|c|}
\hline Combination & Class & Period length \\
\hline \hline$t_{g}^{i}<t_{t}^{j} \wedge t_{t}^{i}>t_{g}^{j}$ & $\mathcal{A}$ & \multirow{2}{*}{$t_{g}^{i}+t_{g}^{j}$} \\
\cline { 1 - 2 }$t_{g}^{i}<t_{t}^{j} \wedge t_{t}^{i}=t_{g}^{j}$ & $\mathcal{B}$ & \\
\cline { 1 - 2 }$t_{g}^{i}<t_{t}^{j} \wedge t_{t}^{i}<t_{g}^{j}$ & $\mathcal{C}$ & \multirow{2}{*}{$t_{g}^{i}+t_{t}^{i}$} \\
\cline { 1 - 2 }$t_{g}^{i}=t_{t}^{j} \wedge t_{t}^{i}=t_{g}^{j}$ & $\mathcal{D}$ & \\
\cline { 1 - 2 }$t_{g}^{i}=t_{t}^{j} \wedge t_{t}^{i}<t_{g}^{j}$ & $\mathcal{E}$ & \multirow{2}{*}{$t_{t}^{j}+t_{t}^{i}$} \\
\hline$t_{g}^{i}>t_{t}^{j} \wedge t_{t}^{i}<t_{g}^{j}$ & $\mathcal{F}$ & \\
\hline
\end{tabular}

4.3. Pure strategies. First, we are going to look for a pure weak Nash equilibrium (pwNE). We recall the definition of the pwNE: a strategy profile $\left(\mathfrak{s}_{i}{ }^{\star}, \mathfrak{s}_{j}{ }^{\star}\right)$ is a pwNE if any $\mathfrak{s}_{i}$ and $\mathfrak{s}_{j}$ are deterministic strategies and verify

$$
\begin{aligned}
u_{i}\left(\mathfrak{s}_{i}, \mathfrak{s}_{j}{ }^{\star}\right) \leq u_{i}\left(\mathfrak{s}_{i}{ }^{\star}, \mathfrak{s}_{j}{ }^{\star}\right), & \forall \mathfrak{s}_{i}, \\
u_{j}\left(\mathfrak{s}_{i}{ }^{\star}, \mathfrak{s}_{j}\right) \leq u_{j}\left(\mathfrak{s}_{i}{ }^{\star}, \mathfrak{s}_{j}{ }^{\star}\right), & \forall \mathfrak{s}_{j} .
\end{aligned}
$$

To help analyse this problem, we introduce the graphical representation of a strategy payoff as pictured in Fig. 3. Referring to strategy $\mathfrak{s}_{i}$, if we represent parameter $t_{g}^{i}$ varying on the horizontal axis and parameter $t_{t}^{i}$ varying on the vertical axis, we can map, on the XY plane, the payoff $u_{i}\left(\mathfrak{s}_{i}, \mathfrak{s}_{j}\right)$ of strategy $\mathfrak{s}_{i}$ against a fixed strategy $\mathfrak{s}_{j}$. We divide the quadrant in zones according to the class the pair $\left(\mathfrak{s}_{i}, \mathfrak{s}_{j}\right)$ belongs to, as defined in Table 2. In each zone, we also represent the corresponding expression of $u_{i}\left(\mathfrak{s}_{i}, \mathfrak{s}_{j}\right)$. Since classes $\mathcal{B}, \mathcal{C}$ and $\mathcal{E}$ are the ones where relations are not symmetrical, we use the same letter but with a prime (e.g., $\left.\mathcal{B}^{\prime}\right)$ to distinguish whether the player with strategy $\mathfrak{s}_{i}$ is the top or bottom player in Table 1 (a prime denotes the bottom player).

The rationale for using the XY plane to look for pure Nash equilibria is as follows:

1. If we plot strategy $\mathfrak{s}_{i}$ payoff against $\mathfrak{s}_{j}$ in the $\mathrm{XY}$ plane, we only have to look for strategy $\mathfrak{s}_{i}{ }^{\star}$ with the highest payoff. That is to say, $\mathfrak{s}_{i}{ }^{\star}$ is the best response to $\mathfrak{s}_{j}$.

2. Next, we select $\mathfrak{s}_{i}{ }^{\star}$ as the new fixed strategy, and we look in the XY plane for the best response, $\mathfrak{s}_{j}{ }^{\star}$. If this strategy is equal to the first fixed strategy, $\mathfrak{s}_{j}$, then $\left(\mathfrak{s}_{i}{ }^{\star}, \mathfrak{s}_{j}{ }^{\star}\right)$ is a pure Nash equilibrium.

A Nash equilibrium found by this method may be weak if we find multiple best responses in any of the previous steps.

4.4. Finding the best responses. To find $\mathfrak{s}_{i}{ }^{\star}$, the best response to $\mathfrak{s}_{j}$, we can eliminate zones $\mathcal{C}^{\prime}, \mathcal{B}, \mathcal{D}, \mathcal{E}^{\prime}$ and
$\mathcal{F}$ in Fig. 3 since their payoff is lower than or equal to a payoff of a neighbouring zone (represented in Fig. 3 by signs $\geq$ and $\vee$ ). We can further restrict the search by selecting the best candidates in the four remaining zones, $\mathcal{A}, \mathcal{B}^{\prime}, \mathcal{C}$ and $\mathcal{E}$. The best response is one of these candidates.

$\mathcal{B}^{\prime}$ All strategies in this zone, $t_{t}^{i}>t_{g}^{j}$, have the same payoff. So, every one of them fares equally well. The complete characterisation of the best candidates in $\mathcal{B}^{\prime}$ is 4

$$
\mathfrak{s}_{i}=\left(t_{t}^{j}, t_{t}^{i}\right) \quad \text { with } \quad t_{t}^{i}>t_{g}^{j} .
$$

$\mathcal{A}$ The best candidates in this zone are strategies with $t_{g}^{i}$ as high as possible (up to $t_{t}^{j}-1$ ) since the payoff is strictly increasing with this parameter. By definition, zone $\mathcal{A}$ has $t_{g}^{i}<t_{t}^{j}$ (see the horizontal axis). Therefore the best candidates must have $t_{g}^{i}=t_{t}^{j}-1$. This zone is also characterised by $t_{t}^{i}>t_{g}^{j}$ (see the vertical axis), but $t_{t}^{i}$ does not influence the payoffs. Therefore, the best candidate strategies in zone $\mathcal{A}$ are

$$
\mathfrak{s}_{i}=\left(t_{t}^{j}-1, t_{t}^{i}\right) \quad \text { with } \quad t_{t}^{i}>t_{g}^{j} .
$$

$\mathcal{C}$ In this zone the payoff is also strictly increasing in parameter $t_{g}^{i}$ (up to $t_{t}^{j}-1$ ). To maximise the payoff, we must increase $t_{g}^{i}$ to its maximum value in $\mathcal{C}$ $\left(t_{g}^{i}=t_{t}^{j}-1\right)$. As for parameter $t_{t}^{i}$, the payoff may increase, level or decrease, depending on the sign of the numerator, $t_{g}^{i}+b_{g}-c_{p t}$, being negative, zero or positive, respectively. If this value is zero or negative, then the payoff is zero or negative and we can discard this zone, as zone $\mathcal{A}$ is better, having strictly positive payoffs. With a positive numerator, we must decrease $t_{t}^{i}$ to its minimum $\left(t_{t}^{i}=1\right)$ in order to maximise the payoff. Thus, the best candidate in zone $\mathcal{C}$ is

$$
\mathfrak{s}_{i}=\left(t_{t}^{j}-1,1\right) \quad \text { when } \quad t_{t}^{j}-1+b_{g}-c_{p t}>0,
$$

otherwise the best response to $\mathfrak{s}_{j}$ is in zone $\mathcal{A}$.

$\mathcal{E}$ In this zone the payoff only depends on parameter $t_{t}^{i}$. Again, it may increase, level or decrease depending on the sign of the numerator, $t_{t}^{j}+b_{g}-c_{p t}-c_{s t}$, being negative, zero or positive, respectively. We can also discard this zone if the numerator is zero or negative. In that case, at least zone $\mathcal{A}$ has a better candidate. With a positive numerator, since $t_{t}^{j}$ is constant the best candidate in zone $\mathcal{E}$ is

$$
\mathfrak{s}_{i}=\left(t_{t}^{j}, 1\right) \text { when } t_{t}^{j}+b_{g}-c_{p t}-c_{s t}>0 .
$$

The identified candidates of the four zones define the set of candidate strategies $\mathfrak{s}_{i}$ from which a best a response to $\mathfrak{s}_{j}$ can be determined. In the next section we show the resulting Nash equilibrium.

\footnotetext{
${ }^{4}$ Recall that $\mathfrak{s}_{i}$ is defined by the pair $\left(t_{g}^{i}, t_{t}^{i}\right)$.
} 


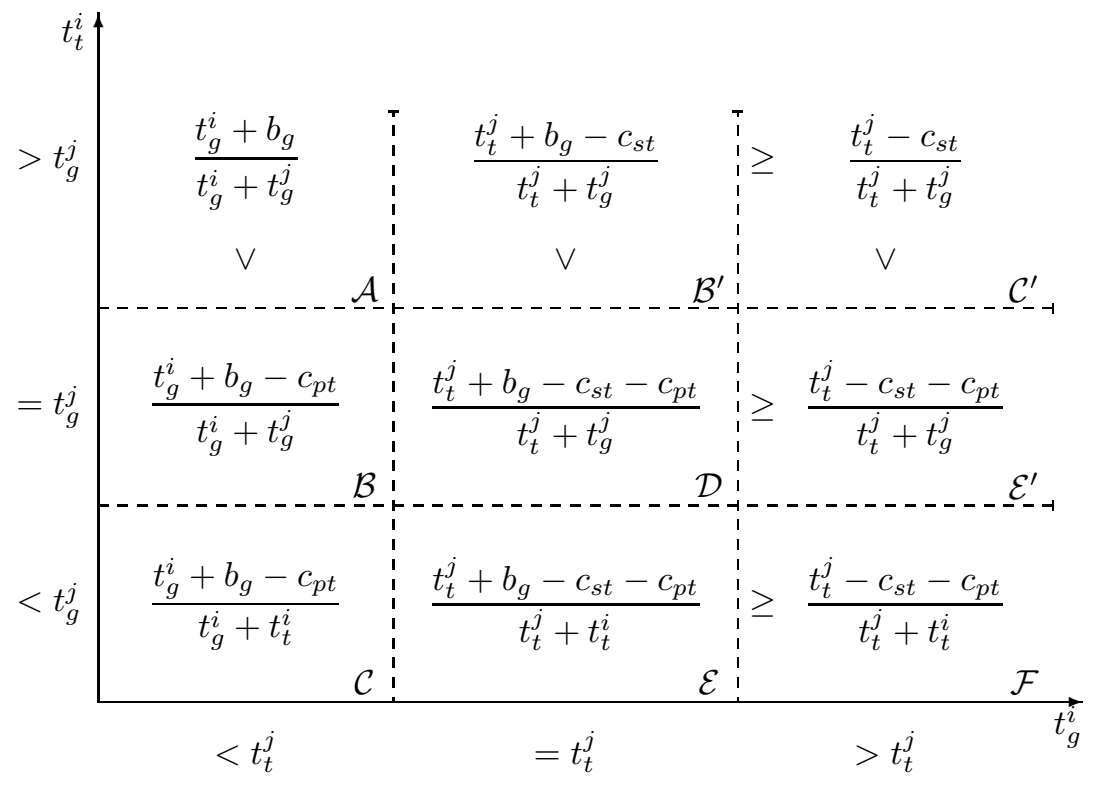

Fig. 3. Graph shows the payoff of player $i, u_{i}\left(\mathfrak{s}_{i}, \mathfrak{s}_{j}\right)$, as a function of strategy $\mathfrak{s}_{i}$ parameters, $t_{g}^{i}$ and $t_{t}^{i}$. The reference values in the graph axis to which these parameters are compared are the parameter values of the opponent player $j, t_{g}^{j}$ and $t_{t}^{j}$. The quadrant is divided into 9 zones according to the class the strategy profile $\left(\mathfrak{s}_{i}, \mathfrak{s}_{j}\right)$ belongs to $(\mathcal{A}$ to $\mathcal{F})$. The labels in the axis represent relations $t_{g}^{i} \gtreqless t_{t}^{j}$ and $t_{t}^{i} \gtreqless t_{g}^{j}$.

4.5. Some properties of Give and Take. Taking into account the analysis in the previous section, there are propositions that can be derived regarding the nature and existence of NEs.

Proposition 1. There is an unbounded number of class $\mathcal{A}$ pwNEs of the form $((1, x),(x-1,2))$ with $x>1$, if $c_{s t} \geq 1 \wedge c_{p t} \geq 1+b_{g}$.

All these profiles are also POs. Although their number is unbounded, they require an unlimitedly patient player as it must wait $x-1$ stages for the resource. For high values of $x$, this can be unrealistic. The proof also shows that there are situations where $c_{s t}<1$ and this strategy profile is still a NE.

This result shows that these equilibria exist if

1. the cost of being punished by action take is high enough, i.e., $c_{s t}$ large enough, meaning greater or equal to the resource value;

2. players are discouraged to take the resource by having a penalty high enough for performing action take, i.e., $c_{p t}$ large enough, meaning greater or equal to the resource value plus bonus.

With this profile, to give the resource is a rational choice. Players receive different payoffs except for $x=$ 2 . In this case, we have strategy profile $((1,2),(1,2))$, which is the single symmetrical profile that verifies this proposition.
Proposition 2. The number of class $\mathcal{A}$ strategy profiles of the form $((x, x+1),(x, x+1))$ that are $p w N E$ s is finite for $c_{p t} \geq 1 \wedge c_{s t} \geq 1 / 2$.

These are the only symmetrical strategy profiles forming a pwNE. This proposition shows that there is a finite number of them. Notice that the single symmetric profile identified in Proposition 1 is a particular case of these strategy profiles. Moreover, for $b_{g}>0$, it is the single PO. If $b_{g}=0$, all of these profiles are POs. The parameters of action take are the ones that play a major role in defining the number of strategy profiles: the higher the values of $c_{p t}$ and $c_{s t}$, the higher the number of symmetrical strategy profiles that exist.

The existence of these profiles raises a problem of coordination similar to the one found in coordination games. These profiles and the ones identified in Proposition 1 have different payoffs, as is also the case in Stag Hunt. As we have said, players facing this coordination dilemma may solve it either before playing or by observing the behaviour of the partner.

Regarding real-life applications, if the penalty for not delivering the book, for not sharing the tractor, for not returning the apartment or for not fulfilling the contract is high enough, then players may be compelled to behave properly. This is similar to the fear of retaliation found in IPD (Axelrod, 1984).

Proposition 3. If $b_{g}=0 \wedge x>c_{p t} \wedge c_{p t}+c_{s t} \geq 1$, there is a $k \in \mathbb{N}$ such that there is an unbounded number of class $\mathcal{C}$ strategy profiles of the form $((x, 1),(y, x+1))$ 
that are pwNEs, with $x>k \wedge y \geq x \wedge y>1$.

These pwNEs occur when the bonus to give the resource is zero. While players may choose a symmetrical cooperative strategy profile, this proposition shows they may turn to asymmetric profiles. In this case, one of the players does nothing, only keeps the resource one stage per period and suffers the cost of being the subject of action take. The other player performs both the give and take actions and thus controls when its partner has the resource. It does not matter if we increase $c_{p t}$, because for all $x>k$ the conditions of this proposition are true, and thus there is an unbounded number of strategy profiles that are pwNEs.

Profiles of this form are not symmetric, in the sense that the two players have different payoffs. Only through simultaneous strategy changes can both players leave these equilibria and achieve payoff equity.

The existence of these pwNEs contrasts with the games we reviewed earlier. With this profile, the player playing strategy $(x, 1)$ controls when the partner has the resource. Even then, the player only relinquishes it for just one stage during the duration of the action period. The partner has no incentive to deviate from its strategy, which amounts to doing nothing at any stage. It has no incentive to give the resource because the bonus is zero, and it has no incentive to take the resource because it gets a lower payoff due to $c_{p t}$. This behaviour is not found in any of the games we reviewed earlier.

Proposition 4. Classes of strategy pairs (or profiles) can be ordered using as sorting criteria Pareto dominance. In the general case, we have the following sequence, where the arrow means that the destination profile Pareto dominates the origin profile:

$$
\mathcal{A} \longleftarrow \mathcal{B} \longleftarrow{ }_{\mathcal{D}}^{\mathcal{C}} \longleftarrow \mathcal{E} \longleftarrow \mathcal{F}
$$

In other words, this proposition states that, for example, for every strategy profile belonging to class $\mathcal{B}$, there is a strategy profile from class $\mathcal{A}$ that Pareto dominates. From the previous propositions, it is clear that a pwNE strategy profile stated in Proposition 3 (class $\mathcal{C}$ ) is Pareto dominated by some strategy profile from class $\mathcal{A}$. However, both players must change their strategies, to go from class $\mathcal{C}$ to class $\mathcal{A}$. To achieve this change simultaneously, players need to establish an agreement. Notice that a strategy profile that is Pareto dominant is not necessarily a Nash equilibrium. Moreover, if players are not cooperative, they may not settle in a Pareto dominant profile. Instead, such players try to exploit their partners.

\section{Numerical analysis}

In this section we show the results of numerical analysis of Give and Take. We used an evolutionary approach, that is to say, a population composed of some strategies was subject to an evolutionary algorithm and we identified the strategies that were more successful. The properties presented in the previous section guide us in choosing the parameters of Give and Take.

5.1. Strategy space. Besides the strategy space introduced in Section 4.1, we defined a strategy with a history of size 1. By observing an action sequence (see, for instance, Table 11), one realises that there are eight possible action combinations. Therefore, a strategy has eight parameters that indicate whether a player should either give the resource or take it, depending on the actions played in the previous stage. The strategy has two additional parameters that indicate what a player should do in the first stage, depending on whether it has the resource or not. Let

$$
s=\left(a_{\mathrm{GT}}, a_{\mathrm{G}-}, a_{+\mathrm{T}}, a_{+-}, a_{\mathrm{TG}}, a_{-\mathrm{G}}, a_{\mathrm{T}+}, a_{-+}, a_{\mathrm{G}}, a_{\mathrm{T}}\right)
$$

represent a strategy where the subscripts in the first eight parameters represent the action played in the previous stage, and the subscripts in the last two parameters represent the action to be performed in the first stage.

This strategy has ten binary parameters and can thus be represented by a natural number from the interval $[0,1023]$. If we assume that doing nothing is encoded by zero and giving or taking (depending on the parameter) is encoded by one, the following equation gives the code of a strategy $s$ :

$$
\begin{aligned}
\operatorname{code}(s)= & a_{\mathrm{GT}}+2 a_{\mathrm{G}-}+2^{2} a_{+\mathrm{T}}+2^{3} a_{+-} \\
& +2^{4} a_{\mathrm{TG}}+2^{5} a_{-\mathrm{G}}+2^{6} a_{\mathrm{T}+}+2^{7} a_{-+} \\
& +2^{8} a_{\mathrm{G}}+2^{9} a_{\mathrm{T}} .
\end{aligned}
$$

5.2. Nice strategies. A general analysis shows that, if a strategy is a best response to others or is able to survive some evolutionary dynamics, it must play nice with itself. Out of 1024 possible strategies, only 194 give the resource. Moreover, it has to play nice with others; namely, the resulting action sequence ideally should belong to class $\mathcal{A}$ independently of who starts with the resource. Out of 523776 possible pairs, only 7517 result in games where strategies give the resource. Therefore, one could sort the 194 strategies according to the number of appearance in the 7517 pairs. On the other hand, one can perform numerical simulations to assess how robust each one of these 194 strategies is against all the other strategies. From this point on, we shall call these 194 strategies nice, alluding to the fact that each one gives the resource when playing against itself.

5.3. Simulations and results. To analyse the fitness of a strategy, we performed a set of simulations in order 
Table 3. Parameters tested in the simulations to evaluate the history-one strategies.

\begin{tabular}{|cl||l|}
\hline$b_{g}$ & bonus to give & $\{0,0.5\}$ \\
$c_{p t}$ & cost perform action take & $\{1.5\}$ \\
$c_{s t}$ & cost perform action take & $\{2.25\}$ \\
$l$ & number of stages & $\{100\}$ \\
& number of generations & 2000 \\
& population size & 100 \\
& mutation probability & $1 \%$ \\
& mutation operator & flip parameter, \\
& & randomisation \\
& selection operator & tournament size 2, \\
& & imitation \\
\hline
\end{tabular}

to see how robust nice strategies are against mutants. We took an evolutionary algorithm approach (Hofbauer and Sigmund, 1998). The chromosome of an individual is just the strategy described in the previous section. In this set of simulations, the initial population was homogeneous. In each generation, all players in the population played with everybody else. The fitness of an individual was the sum of the payoffs it obtained in all the games it participated in. We used two selection operators, namely, tournament selection with size two and imitation. We used two mutation operators, namely, flipping one of the ten strategy parameters or randomisation of all parameters. In each experimental setting, the mutation operator was applied with $1 \%$ probability. Table 3 shows the parameters used in this set of experiments.

For each possible strategy with history of length one we counted the number of individuals per generation using that strategy. That is to say, we created an histogram of all strategies using as data all the generations from the first set of experiments. Figures 4 and 5 show the histograms. Instead of ordering the strategies by their encoding, the horizontal axis is sorted by the number of occurrences of each strategy. Using this view, one can see in Fig. 4 that strategy occurrence follows a power law distribution, meaning most strategies occur rarely, i.e., only in the first generations. Figure 5 shows the top ten strategies in all simulations $\left(b_{g}\right.$ is either 0 or 0.5$)$, in simulations with $b_{g}=0$, and in simulations with $b_{g}=0.5$. Each point in this plot has a label showing the strategy code. If the strategy is not nice, there is an additional star symbol in the label. In this plot, one can see that, in the top ten positions, seven out of the strategies are nice, meaning that it is advantageous to share the resource by giving it.

The next set of results shows properties of the strategies that were on the three top ten ranks. Table 4 shows the properties of these strategies. There are a total of twelve strategies and two thirds are nice. All but one of the nice strategies give the resource right after the partner gives it to them (see column $a_{-G}$ ). Only strategy 584

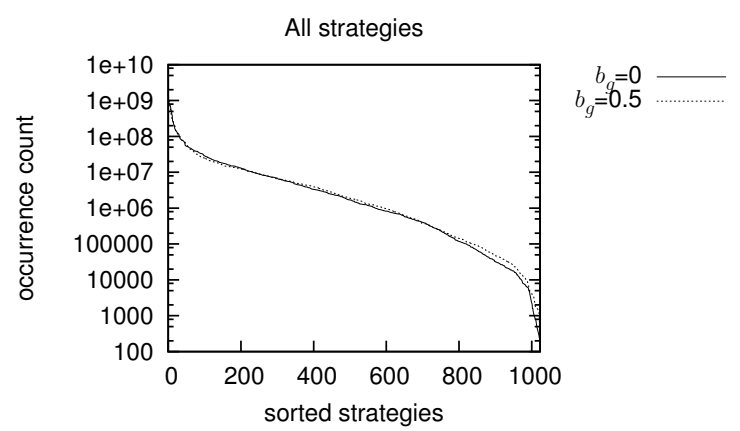

Fig. 4. Results of simulations using the history strategy space. This graph shows all strategies sorted in descending order of their number of occurrences.

keeps the resource and gives it only when both players do nothing. A common characteristic is giving the resource when they have it; columns $a_{\mathrm{TG}}$ through $a_{\mathrm{T}+}$ have $79 \%$ of the give actions. This corresponds to being nice. On the other hand, all but one are spiteful since they take the resource if nothing happened in the previous iteration (see column $a_{-+}$). Again, strategy 584 does not take the resource. They can be considered patient as they do nothing when they lose resource possession. There are only $9 \%$ of the take actions in columns $a_{\mathrm{GT}}$ through $a_{+\mathrm{T}}$.

As for the four strategies that are not nice, they have varying characteristics which are more visible when we observe their action sequences. Strategies 18 and 544 do nothing when playing among themselves. This means that on average they fare as well as nice strategies when $b_{g}$ is zero. The other strategies, 130 and 931, take the resource when playing among themselves. However, when 18 plays with the other top ten strategies, it does nothing, and it keeps the resource twice as many stages as the opponent. This means it has an advantage compared to the opponent. On the other hand, strategy 931 also takes the resource when playing with some of the top strategies and for this reason has a lower rank.

\section{Discussion}

In a Give and Take game with a defined number of stages, $l$, there might be a problem of coordination. This happens in all cases except for $c_{p t}>l$, which means that the loss from the penalty for taking the resource cannot possibly be recovered in the last $l$ stages. If players cooperate, both can profit from bonus $b_{g}$ and avoid the outcome of Nash equilibria that may be lower. This stands even when $b_{g}=0$, due to penalties $c_{p t}$ and $c_{s t}$ that come into play if a player retaliates, meaning that it takes the resource from the partner. The cooperative strategy profile that equally maximises both players' payoffs is the one in which both agents give the resource after holding it for the 


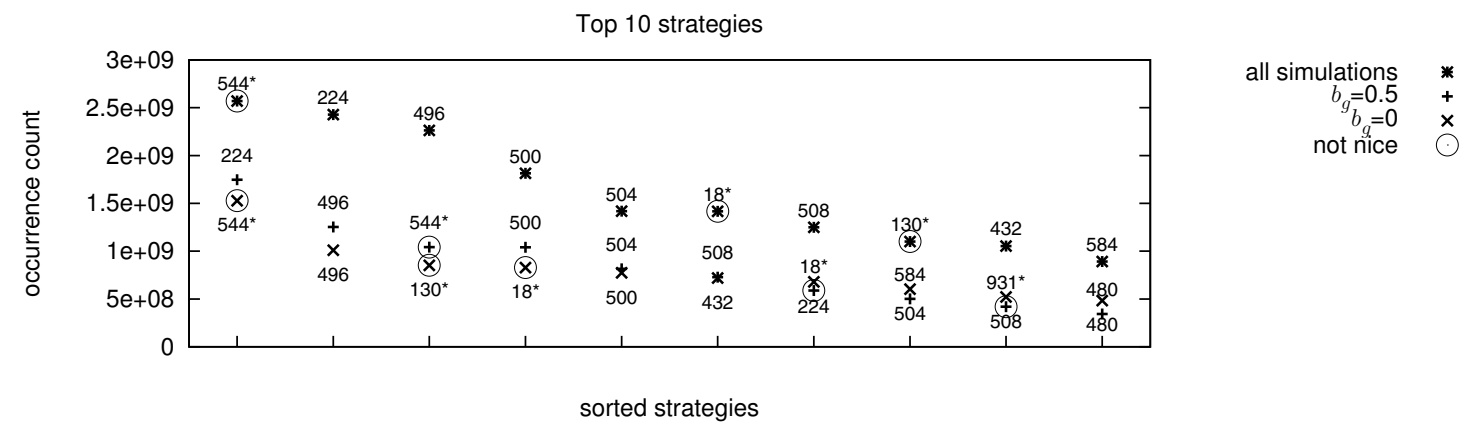

Fig. 5. Results of simulations using the history strategy space. This graph shows top ten strategies sorted by descending order of their number of occurrences.

Table 4. Strategy parameters of those in the three top ten ranks. The first rows show the parameters of strategies that are not nice (marked with a star). The remainder rows are for nice strategies. The three rightmost columns show their place in the three ranks.

\begin{tabular}{|l||cccc|cccc|cc||ccc|}
\hline $\begin{array}{l}\text { strategy } \\
\text { code }\end{array}$ & $a_{\mathrm{GT}}$ & $a_{\mathrm{G}-}$ & $a_{+\mathrm{T}}$ & $a_{+-}$ & $a_{\mathrm{TG}}$ & $a_{-\mathrm{G}}$ & $a_{\mathrm{T}+}$ & $a_{-+}$ & $a_{\mathrm{G}}$ & $a_{\mathrm{T}}$ & \multicolumn{5}{|c|}{ rank } \\
\hline $18^{\star}$ & - & $\mathrm{T}$ & - & + & $\mathrm{G}$ & + & + & - & + & - & 6 & 4 & 7 \\
$130^{\star}$ & - & $\mathrm{T}$ & - & + & + & + & + & $\mathrm{T}$ & + & - & 8 & 3 & 15 \\
$544^{\star}$ & - & - & - & + & + & $\mathrm{G}$ & + & - & + & $\mathrm{T}$ & 1 & 1 & 3 \\
$931^{\star}$ & $\mathrm{T}$ & $\mathrm{T}$ & - & + & + & $\mathrm{G}$ & + & $\mathrm{T}$ & $\mathrm{G}$ & $\mathrm{T}$ & 14 & 27 & 9 \\
224 & - & - & - & + & + & $\mathrm{G}$ & $\mathrm{G}$ & $\mathrm{T}$ & + & - & 2 & 7 & 1 \\
432 & - & - & - & + & $\mathrm{G}$ & $\mathrm{G}$ & + & $\mathrm{T}$ & $\mathrm{G}$ & - & 9 & 6 & 11 \\
480 & - & - & - & + & + & $\mathrm{G}$ & $\mathrm{G}$ & $\mathrm{T}$ & $\mathrm{G}$ & - & 11 & 10 & 10 \\
496 & - & - & - & + & $\mathrm{G}$ & $\mathrm{G}$ & $\mathrm{G}$ & $\mathrm{T}$ & $\mathrm{G}$ & - & 3 & 2 & 2 \\
500 & - & - & $\mathrm{T}$ & + & $\mathrm{G}$ & $\mathrm{G}$ & $\mathrm{G}$ & $\mathrm{T}$ & $\mathrm{G}$ & - & 4 & 5 & 4 \\
504 & - & - & - & $\mathrm{G}$ & $\mathrm{G}$ & $\mathrm{G}$ & $\mathrm{G}$ & $\mathrm{T}$ & $\mathrm{G}$ & - & 5 & 8 & 5 \\
508 & - & - & $\mathrm{T}$ & $\mathrm{G}$ & $\mathrm{G}$ & $\mathrm{G}$ & $\mathrm{G}$ & $\mathrm{T}$ & $\mathrm{G}$ & - & 7 & 9 & 6 \\
584 & - & - & - & $\mathrm{G}$ & + & + & $\mathrm{G}$ & - & + & $\mathrm{T}$ & 10 & 12 & 8 \\
\hline
\end{tabular}

same number of stages. Such a behaviour is the preferred one of Homo Sociologicus (López-Pérez, 2008; Fehr and Gintis, 2007; Gintis, 2000), in opposition to the behaviour of Homo Economicus that would instead select the actions of Nash equilibria. When $b_{g}>0$, Homo Sociologicus' behaviour converges to the optimal profile where agents give the resource right after receiving it. It is optimal from the fact that both players equally divide the resource possession and maximise the bonus by giving it back and forth every stage.

For an undefined number of stages, this game has many different pure weak Nash equilibria. These range from symmetrical, where players have identical strategies, to asymmetrical profiles, where one player does nothing while the other periodically gives and takes the resource.

Players that avoid exploitative strategies settle in symmetric strategy profiles. This behaviour would be favoured by Homo Sociologicus. To achieve that, players have to negotiate, either explicitly, by communication and agreement, or implicitly, by self-adapting their strategies while playing. The rational result of negotiation is the profile $((1,2),(1,2))$ because it is the single symmetrical
PO of this game for $b_{g}>0$, besides also being a pwNE (see Propositions 1 and 2). When the bonus for giving the resource, $b_{g}$, goes down to zero, an unbounded number of symmetric POs appear in addition to that one.

The costs of the take action, $c_{p t}$ and $c_{s t}$, can be considered penalties that serve as deterrents of noncooperative behaviour. It should be noted that these two parameters also serve to establish a dilemma in the game. If both are zero, there is no dilemma and the player without the resource always takes it.

Propositions 1 and 2 show that if take costs are sufficiently high, cooperation is a NE. Although there are unlimited asymmetric cooperative profiles (Proposition 1), there are also some symmetric ones (Proposition 2). Proposition 3 showed that if there is no incentive to give the resource $\left(b_{g}=0\right)$, one player does nothing during the game. It only has the resource when the partner gives it to it.

The analysis done in Section 4 focused on a subset of the entire strategy space. On the other hand, the analysis done in Section 5 focused on all strategies with history size equal to one. The results showed that the most 
successful strategies are those that share the resource by giving it right after receiving, followed by strategies that do nothing. Moreover, when there is a bonus for giving the resource, strategies that give the resource rise to the first position.

The propositions and experimental work show that the fear of retaliation and positive recognition of those who share are important to promote cooperation in Give and Take as well as in real world scenarios it models. This means that people return a book to the library, a family returns a holiday facility, and a farmer returns a tractor.

These are some of the real scenarios that are better modelled by Give and Take. The asymmetry of Give and Take is a realistic feature that PGP does not account for, although the latter has been used in cases where players have different roles with different bargaining powers (Velez et al., 2009). Give and Take is best suited for modelling turn-taking scenarios (Lau and Mui, 2012). Ownership of the resource and the possibility to give it or having it taken may help model access a common pool resource such as a fishery (Fehr and Leibbrandt, 2011; Jones, 2006), water sources (Wallace et al., 2003; Rowland, 2005) or forests. This asymmetry is also present in entities that share an international river. The nation upstream may use the water for producing electricity or irrigation, while a downstream nation is left with a lower resource value. Even within a country, a dam may restrict water access to some citizens while improving the benefits of others (Wallace et al., 2003).

Further applications of Give and Take are possible if we consider extensions to the game. A political system dominated by two parties (Ward, 1998) can be modelled by Give and Take. In this case, the resource represents the government. The extension consists of the player with the resource having a third action: drop the resource. In this application, dropping the resource is only done at specific turns. After dropping the resource, parties invest in campaigning to gain the favour of the electorate. Taking the resource is equivalent to the player in the opposition promoting social unrest, proposing a motion of censure or capitalising on a general strike. Another application of a multi-player Give and Take is to a multi-party system.

Traffic flow in intersections without semaphores (Dong and Dai, 2011) can also be modelled by a variation of Give and Take. At certain intersections, only the cars arriving from a specific lane can use the intersection to turn left. In this application, the resource is access to the intersection. The extension is the fact that the player that has the resource may leave the game and hand the resource to a new player. This extension may be well suited to model the behaviour of drivers at intersections (Doniec et al., 2008).

\section{Conclusions}

We have analysed the Give and Take game, where two players hold a common resource in turns, each of these defining a stage of the game, with different roles for both players. The player with the resource can give it to its partner, while the player without the resource can take it from its partner. It was shown that this game has numerous pure weak Nash equilibria and PO profiles. This is a unique feature of this game which contrasts with common games such as PD, Ultimatum, PGP and Centipede, or their iterated versions. The fact that there are many pure weak Nash equilibria and PO profiles raises a problem of coordination as found in games such as Battle of Sexes. In Give and Take, players may coordinate while playing the game, by mutual adaptation to the partner's strategy.

Another unique feature is that the take action may be seen as a built-in punishment for non-cooperative behaviour. Knowing this, an individual may be induced to cooperate, fearing retaliation costs. Moreover, neither cooperation induction, nor role changing nor coordination do require any entity besides the players. All these make Give and Take an interesting tool to study agreements and their subsequent fulfilment or breaking.

There are social situations that are better represented by Give and Take than by previous games. It is more adequate to model sharing of a durable and indivisible resource by two individuals without external intervention, i.e., in a self-organised process. We have given examples of book lending in a library, community tractor sharing, holiday flat sharing, and service contracts. In these scenarios, at each time instant only one person can read the book, only one farmer can plough the fields, only one family can use the premises, and only one individual can provide the service at each stage.

In this paper, we have studied Give and Take divided in two cases: undefined and defined number of stages. In the first case, $\mathrm{PO}$ and pwNE strategy profiles range from symmetric to asymmetric. In symmetric profiles both players give the resource and never take, while in asymmetric profiles one player plays both the give and take actions while the other remains passive. The higher the costs of taking the resource, the greater the number of symmetric profiles, which means that punishing favours cooperative behaviour. In the case of a defined number of stages, simulations show that strategies that give the resource rank higher compared to strategies that take the resource. Even if there is no bonus for giving the resource, strategies that give obtain high ranks.

Give and Take shows a richness of choices and dilemmas that players are faced with in both situations. Equity exists if both players cooperate and share the resource in equal terms. There are different ways to achieve such a cooperation level. Players may establish agreements, for instance, by direct negotiation or by 
mutual strategy adaptation along stages.

The wide choice of cooperative strategies provides a rich environment for the study of negotiation, agreement, treason, retaliation and norm enforcement. We intend to explore these situations using Give and Take as a supporting tool. Also concerning future developments, we plan to increase the number of players and resources.

With more than two players, there is the possibility of coalitions, where teams of players compete for the resource. In terms of strategy space, this results in an increase in complexity. With $n$ players in a game, a player can give the resource to the $n-1$ players. As for taking the resource, we have to deal with the possibility of more than one player taking the resource, meaning that we have to decide who gets the resource in the next stage of the game.

With more than one resource, there is an increased resource exchange strategy space. Will a player never give a resource to someone that has already one resource? Will a player take a resource from someone that has more than one? We also intend to study the problem of treason and its avoidance or control under this extended Give and Take game model.

\section{Acknowledgment}

We thank J.M. Castro Caldas for suggesting the service contract example, Fernando Ramos for comments to improve the organisation of the paper, and Rob Mills for proofreading.

This work was partially supported by FCT, Portugal (UID/Multi/04046/2013).

\section{References}

Akiyama, E. and Kaneko, K. (2000). Dynamical systems game theory and dynamics of games, Physica $D$ 147(3-4): 221-258.

Anderlini, L. (1999). Communication, computability, and common interest games, Games and Economic Behavior 27(1): 1-37.

Axelrod, R. (1984). The Evolution of Cooperation, Basic Books New York, New York, NY.

Binmore, K. (1996). A note on backward induction, Games and Economic Behavior 17(1): 135-137.

Blackwell, C. and McKee, M. (2003). Only for my own neighborhood? Preferences and voluntary provision of local and global public goods, Journal of Economic Behavior \& Organization 52(1): 115-131.

Boyd, R., Gintis, H., Bowles, S. and Richerson, P.J. (2003). The evolution of altruistic punishment, Proceedings of the $\mathrm{Na}$ tional Academy of Sciences 100(6): 3531-3535.

Brembs, B. (1996). Chaos, cheating and cooperation: Potential solutions to the prisoner's dilemma, Oikos 76(1): 14-24.
Camerer, C. (2003). Behavioral Game Theory, Princeton University Press, Princeton, NJ.

Cason, T.N. and Mui, V.-L. (1998). Social influence in the sequential dictator game, Journal of Mathematical Psychology 42(2-3): 248-265.

Chen, X. and Deng, X. (2006). Settling the complexity of two-player Nash equilibrium, 47th Annual IEEE Symposium on Foundations of Computer Science, FOCS'06, Berkeley, CA, USA, pp. 261-272.

Dong, H. and Dai, Z. (2011). A multi intersections signal coordinate control method based on game theory, 2011 International Conference on Electronics, Communications and Control (ICECC), Ningbo, China, pp. 1232-1235.

Doniec, A., Mandiau, R., Piechowiak, S. and Espié, S. (2008). A behavioral multi-agent model for road traffic simulation, Engineering Applications of Artificial Intelligence 21(8): 1443-1454.

Fehr, E. and Gächter, S. (2002). Altruistic punishment in humans, Nature 415(6868): 137-140.

Fehr, E. and Gintis, H. (2007). Human motivation and social cooperation: Experimental and analytical foundations, $\mathrm{An}$ nual Review of Sociology 33: 43-64.

Fehr, E. and Leibbrandt, A. (2011). A field study on cooperativeness and impatience in the tragedy of the commons, Journal of Public Economics 95(9-10): 1144-1155.

Gintis, H. (2000). Game Theory Evolving-A Problem-centered Introduction to Modeling Strategic Interaction, 1st Edn., Princeton University Press, Princeton, NJ.

Helbing, D., Schönhof, M., Stark, H.-U. and Holyst, J.A. (2005). How individuals learn to take turns: Emergence of alternating cooperation in a congestion game and the prisoner's dilemma, Advances in Complex Systems 8(1): 87-116.

Hofbauer, J. and Sigmund, K. (1998). Evolutionary Games and Population Dynamics, Cambridge University Press, Cambridge.

Jones, P.J.S. (2006). Collective action problems posed by no-take zones, Marine Policy 30(2): 143-156.

Koller, D., Megiddo, N. and von Stengel, B. (1996). Efficient computation of equilibria for extensive two-person games, Games and Economic Behavior 14(2): 247-259, http://www.sciencedirect.com/science/ article/pii/s0899825696900512

Lau, S.-H. and Mui, V.-L. (2012). Using turn taking to achieve intertemporal cooperation and symmetry in infinitely repeated $2 \times 2$ games, Theory and Decision 72(2): 167-188, DOI: 10.1007/s11238-011-9249-4.

López-Pérez, R. (2008). Aversion to norm-breaking: A model, Games and Economic Behavior 64(1): 237-267.

Mariano, P. and Correia, L. (2002a). The effect of agreements in a game with multiple strategies for cooperation, in R. Standish, M.A. Bedau and H.A. Abbass (Eds.), Artificial Life VIII: Proceedings of the Eighth International Conference on Artificial Life, MIT Press, Cambridge, MA, pp. 375-378. 
Mariano, P. and Correia, L. (2002b). A game to study coordination and cooperation, 5th Workshop on Deception, Fraud and Trust in Agent Societies, Bologna, Italy, pp. 101-112.

Mariano, P. and Correia, L. (2003). A resource sharing model to study social behaviours, Progress in Artificial Intelligence-11th Portuguese Conference on Artificial Intelligence, EPIA 2003, Beja, Portugal, pp. 84-88.

McCarter, M.W., Budescu, D.V. and Scheffran, J. (2011). The give-or-take-some dilemma: An empirical investigation of a hybrid social dilemma, Organizational Behavior and $\mathrm{Hu}$ man Decision Processes 116(1): 83-95.

McKelvey, R.D. and Palfrey, T.R. (1992). An experimental study of the centipede game, Econometrica 60(4): 803-836.

Nicolò, A. and Yu, Y. (2008). Strategic divide and choose, Games and Economic Behavior 64(1): 268-289.

Nowak, M., Bonhoeffer, S. and May, R. (1994). Spatial games and the maintenance of cooperation, Proceedings of the National Academy of Sciences 91(11): 4877-4881.

Ottone, S. (2008). Are people Samaritans or Avengers?, Economics Bulletin 3(10): 1-3.

Papadimitriou, C.H. (1994). On the complexity of the parity argument and other inefficient proofs of existence, Journal of Computer and System Sciences 48(3): 498-532. http://www.sciencedirect.com/science/ article/pii/s0022000005800637

Rosenthal, R.W. (1981). Games of perfect information, predatory pricing and the chain-store paradox, Journal of Economic Theory 25(1): 92-100, http://ideas.repec.org/a/eee/jetheo/ v25y1981i1p92-100.html

Rowland, M. (2005). A framework for resolving the transboundary water allocation conflict conundrum, Ground Water 43(5): 700-705.

Shapley, L.S. (1953). Stochastic games, Proceedings of the National Academy of Sciences 39(10): 1095-1100, http://www.pnas.org/content/39/10/ 1095.short

Shoham, Y. and Leyton-Brown, K. (2009). Multiagent Systems: Algorithmic, Game-theoretic and Logical Foundations, Cambridge University Press, Cambridge.

Sigmund, K., Hauert, C. and Nowak, M.A. (2001). Reward and punishment in minigames, Proceedings of the National Academy of Sciences 98(19): 10757-10762.

Sutter, M. and Strassmair, C. (2009). Communication, cooperation and collusion in team tournaments-an experimental study, Games and Economic Behavior 66(1): 506-525.

van Dijk, F., Sonnemans, J. and van Winden, F. (2002). Social ties in a public good experiment, Journal of Public Economics 85(2): 275-299.

Velez, M.A., Stranlund, J.K. and Murphy, J.J. (2009). What motivates common pool resource users? Experimental evidence from the field, Journal of Economic Behavior \& Organization 70(3): 485-497.
Wallace, J.S., Acreman, M.C. and Sullivan, C.A. (2003). The sharing of water between society and ecosystems: from conflict to catchment-based co-management, Philosophical Transactions of the Royal Society of London, Series B: Biological Sciences 358(1440): 2011-2026.

Ward, H. (1998). A game theoretic analysis of the politics of taking it in turns, British Journal of Political Science 28(2): 355-387.

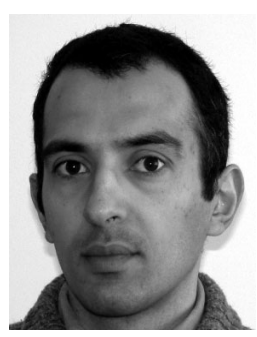

Pedro Mariano is an invited assistant professor at the University of Lisbon. His research interests are focused on the evolution of cooperation using the Give-Take game, in which agent capabilities promote population sustainability, and on developing agents capable of playing multi-player games such as Diplomacy. He has published in major international conference proceedings. He has participated in international research projects as a team member.

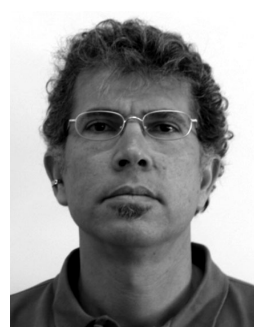

Luís Correia is an associate professor with habilitation at the Department of Informatics of the Faculty of Sciences of the University of Lisbon (DI-FCUL). Since 2004 he has led the Laboratory of Agent Modelling (LabMAg) research centre. Currently he is the head of a department at the DI-FCUL. His research interests are in the area of artificial life, autonomous robots and selforganisation in multi-agent systems. Besides lecturing in the three cycles of informatics, he has also appointments at post-graduations in cognitive science and in complexity sciences (www.di.fc.ul.pt/ lcorreia).

Received: 23 July 2014

Revised: 29 January 2015

Re-revised: 21 March 2015 